\title{
Sarcoidosis presenting as orbital pseudotumor
}

\author{
S. Kobak ${ }^{1}$, A. Topaloglu², G. Öncel ${ }^{3}$, A. Karaarslan ${ }^{4}$ \\ ${ }^{1}$ Department of Rheumatology; '2Department of Ophthalmology; \\ ${ }^{3}$ Department of Radiology; ${ }^{4}$ Department of Ortopedics, Sifa University, Faculty of Medicine, Izmir, Turkey
}

\begin{abstract}
SUMMARY
Sarcoidosis is a systemic multiorgan disorder of unknown etiology characterized by a non-caseating granuloma reaction. Ocular involvement has been reported in $25-60 \%$ of the patients. Seven percent of the patients with sarcoidosis may first see an ophthalmologist due to ocular complaints.

This report aims to present our diagnostic and treatment approach to a female patient with significant unilateral lacrimal gland swelling and musculoskeletal involvement, who was diagnosed with sarcoidosis on the basis of tests and histological studies.
\end{abstract}

Key words: Sarcoidosis; orbital pseudotumor; presentation.

Reumatismo, 2015; 67 (2): 78-81

\section{INTRODUCTION}

Carcoidosis is a systemic multi-organ $\checkmark$ disorder of unknown etiology, characterized by a non-caseating granuloma (1). Although its pathogenesis is not clear, a non-specific inflammatory response and immune system reaction occur due to some genetic and environmental factors (2). Pro-inflammatory cytokines secreted by Th1 cells and macrophages trigger an inflammatory cascade and tissue permeability, then cellular influx and local cell proliferation which result in the formation of a granuloma (3).

A non-caseating epithelioid cell granuloma is the main pathological evidence of sarcoidosis (4). Variations in clinical manifestations, course and prevalence among different races and ethnic groups confirm the heterogeneity of this disease (5). Sarcoidosis is more likely to occur among females in the fifth decade of life. It is a chronic granulomatous disease with various clinical presentations (6). The most common are pulmonary infiltrations, enlarged hilar lymph nodes, skin and eye lesions. Non-caseating granuloma is a pathognomonic histological feature. These granulomas may occur in almost any or- gan in the body. Ocular involvement has been reported in $25-60 \%$ of the patients (7). Seven percent of the patients with sarcoidosis may first see an ophthalmologist with complaints of ocular involvement (8). Anterior uveitis is the most common ocular manifestation, followed by posterior uveitis which may have severe impacts on visual acuity (9).

Rare ocular manifestations include the involvement of the lacrimal gland and ocular tissues other than the lacrimal gland and the optic nerve. Histological and scintigraphic studies have revealed a high prevalence of asymptomatic conjunctiva and lacrimal gland involvement in sarcoidosis (10). This report is aimed to present our diagnostic and treatment approach to a female patient with a significant unilateral lacrimal gland swelling and musculoskeletal involvement, who was diagnosed with sarcoidosis on the basis of tests and histological studies.

\section{CASE REPORT}

A 24 year old female patient was referred to our clinic with complaints of pain, swelling and limited range of motion in both ankles, dry cough, erythema nodosum 
and left upper eyelid swelling. Her medical history showed that she came to our hospital due to gradually increasing complaints over the last 3 months. She complained mainly left eyelid swelling, but previously she also had joint pain and erythema nodosum. The physical examination identified bilateral ankle synovitis, pretibial skin lesions of erythema nodosum and left eyelid swelling. As to laboratory tests, the erythrocyte sedimentation rate was $48 \mathrm{~mm} / \mathrm{h}$ and the C-reactive protein level was $5.2 \mathrm{mg} / \mathrm{dL}$ (reference range: $0-5 \mathrm{mg} / \mathrm{dL}$ ). The results of the rheumatoid factor and anti-nuclear antibody (ANA) measurement were within the normal range and anti-CCP and ANCA were negative. Thyroid function tests, liver function tests and kidney function tests were within normal limits. Routine urinalysis was normal. Abdominal ultrasound did not show any abnormal findings. Chest $\mathrm{X}$ ray showed a bilateral hilar fullness. Torax computed tomography (CT) detected bilateral enlarged hilar lymph nodes, the largest of which had a 2-cm diameter (Fig. 1). The level of serum angiotensin converting enzyme (ACE) was $118 \mathrm{mg} / \mathrm{dL}$ (reference value $<56 \mathrm{mg} / \mathrm{dL}$ ). Serum calcium and vitamin hydroxy D3 levels were within the normal range. The chest disease specialist performed a biopsy guided by endobronchial ultrasonography (EBUS) of the enlarged hilar lymph nodes. The non-caseating granulomas were found to be consistent with sarcoidosis. The patient was seen by our consultant ophthalmologist due to the swollen eye. The ophthalmologic examination of the patient revealed a swelling in the distal third of the upper left eyelid, which was not associated with erythema of the overlying skin.

The mass was mobile and easily distinguishable from the surrounding tissue. A swelling of the lacrimal gland attracted attention when the left upper eyelid was averted. Interpalpebral opening was 8 $\mathrm{mm}$ on both the right and the left sides. Eye movements were free in every direction. The eyes were near and orthophoric at distance. Corrected visual acuity was full in the right eye and 0.2 in the left eye. The cause of low vision in the left eye was found to be anisometropic amblyopia. The refractive error was not detected in the right eye, while it was +5.00 diopters in the left eye. Intraocular pressure was measured using an applanation tonometer and was found to be $17 \mathrm{mmHg}$ in both eyes. The biomicroscopic examination of the right and left anterior segments did not reveal any abnormal findings. The Schirmer score (test 1 without anesthesia) was 16 $\mathrm{mm}$ on the right side and $11 \mathrm{~mm}$ on the left side. No pathological finding was detected during fundoscopy. Magnetic resonance

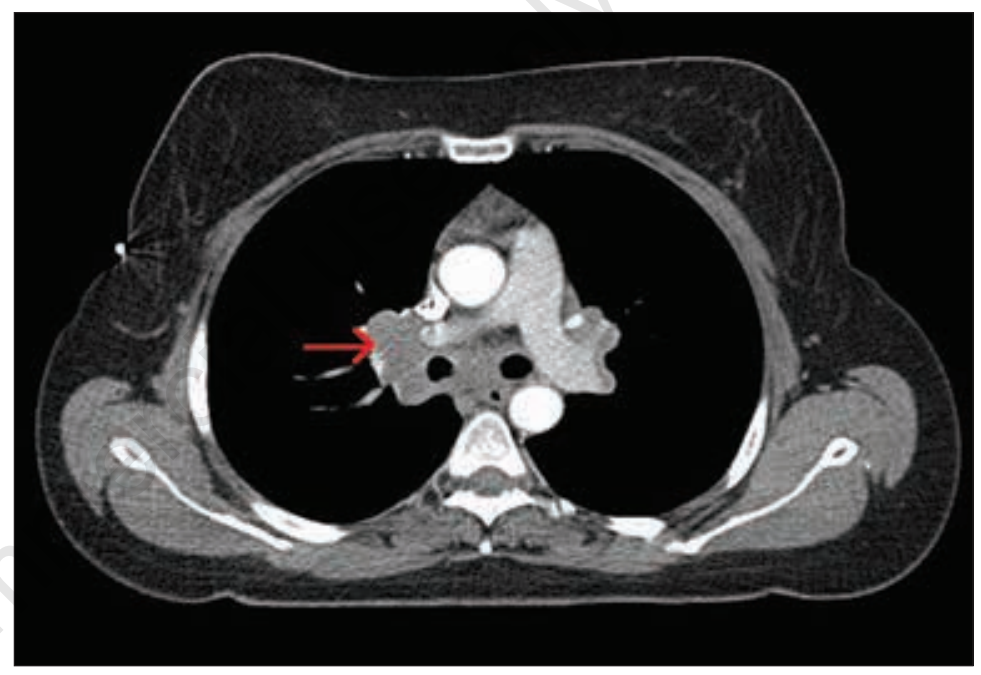

Figure 1 - Torax computed tomography revealed bilateral enlarged hilar and mediastinal lymph nodes.

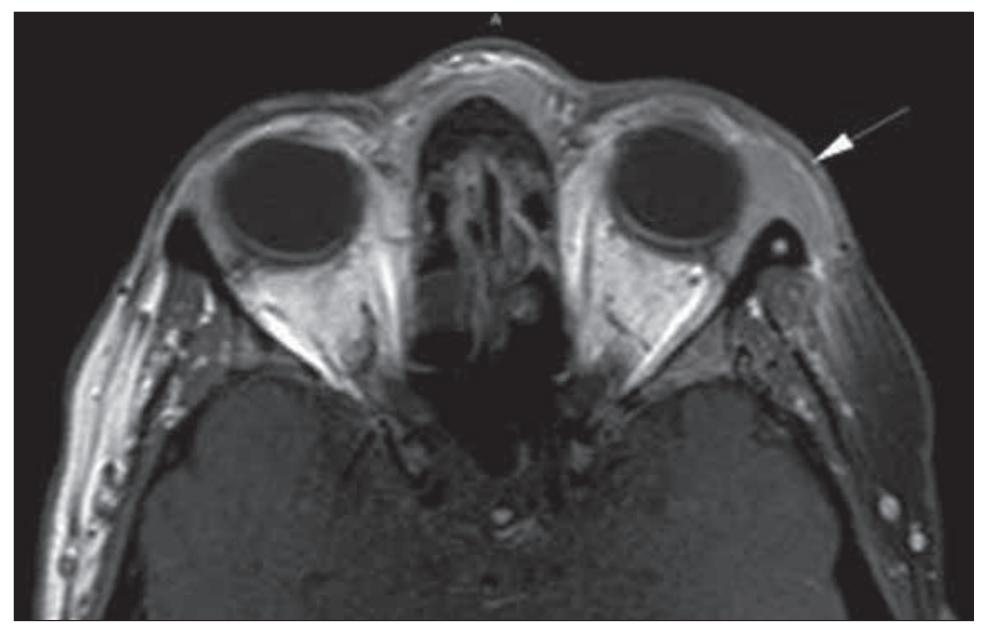

Figure 2 - Orbita magnetic resonance imaging showed an enlargement of the lacrimal gland located in the upper temporal portion of the left orbita without bone destruction. 
(MR) scans of the orbita showed that the enlargement of the lacrimal gland was in the upper temporal portion of the left orbita and was not associated with bone destruction (Fig. 2). The right lacrimal gland was normal. The inflammatory reaction in the left lacrimal gland was considered to be a manifestation of sarcoidosis. The diagnosis of sarcoidoisis was made on the basis of an EBUS-guided biopsy. No lacrimal gland biopsy was performed. Oral corticosteroid therapy was started at a dose of 1 $\mathrm{mg} / \mathrm{kg}$ daily. Weekly follow up visits were scheduled in the first month of therapy and a lacrimal gland biopsy was considered if no clinical improvement was achieved. At 3 months, the patient had no musculoskeletal complaints and laboratory findings (erythrocyte sedimentation rate, C-reactive protein) went back to normal. An assessment of the affected eye showed a significant reduction in the size of the mass lesion. At 6 months into treatment, both lacrimal glands were within the normal range in the MR scans of the orbita. Meanwhile CT scans of the chest revealed a significant regression in the size of the enlarged hilar lymph nodes. It was planned to gradually reduce the doses of oral steroids. The patient is still followed up in an outpatient setting.

\section{DISCUSSION AND CONCLUSIONS}

Sarcoidosis is a Th1-mediated, multi-system, multi-organ granulomatous disease of unknown etiology. Bilateral enlarged hilar lymph nodes, pulmonary infiltrations, skin and ocular lesions are the most common manifestations of this disease.

Ocular involvement in sarcoidosis may affect various portions of the eye, such as optic nerve, blood vessels, extraocular muscles, orbita, uvea/retina. It may, therefore, present with a wide spectrum of clinical signs and symptoms. In ocular sarcoidosis, keratoconjunctivitis sicca and punctuate epithelitis may occur as a result of the involvement of the lacrimal gland.

The affected anterior segment may lead to conjunctival granulomas, conjunctivitis, scleritis, episcleritis, interstitial keratitis and anterior uveitis. The granulomatous inflammation of the conjunctiva is a common manifestation of sarcoidosis (11). These nodules are multiple, pale yellow, translucent, non-caseating granulomas and may be the first sign of sarcoidosis. The most common manifestation of ocular sarcoidosis is anterior uveitis which is present in $60 \%$ of the cases. Typical findings of anterior uveitis include keratic precipitates, cells and flare in the anterior camera, Busacca and Koeppe nodules, anterior and posterior synechia, and iris granuloma. Uveitis may not always be associated with symptoms such as photophobia, lacrimation and redness.

In such conditions, persistent eye damage may occur secondary to the silent uveitis. Although intermediary uveitis is relatively less common, snowball opacities in the vitreous cavity may suggest sarcoidosis. Posterior uveitis occurs in $30 \%$ of patients and $12 \%$ of them develop choroidal granuloma (12). Nearly half of the patients with ocular sarcoidosis have not been previously diagnosed with systemic sarcoidosis and present with ocular complaints as first manifestation of the disease.

However, there is a strong correlation between orbital sarcoidosis and systemic sarcoidosis.

A diagnosis of systemic sarcoidosis was made by Colison et al. in 14 out of 15 patients with orbital sarcoidosis (13). Our patient was diagnosed with systemic sarcoidosis on the basis of test and biopsy results. While systemic sarcoidosis is more likely to occur in the 2nd and 3rd decades, orbital sarcoidosis tend to occur in women in the sixth decade of life. Henderson reported that the average age of these patients is 57 (14). Peterson et al. also described 2 elderly patients with orbital sarcoidosis (15). On the contrary, our patient was a 24 year-old female and became the youngest patient with sarcoidosis ever reported in the medical literature. The first cases of orbital sarcoidosis were reported by Holm and King $(16,17)$. Benedict et al. reviewed 1000 cases of primary orbital tumor and found only two cases of orbital 
sarcoidosis (18). This disease sarcoidosis may present with rapidly progressive pseudo-tumor, exophtalmus and/or limitation of eye movements and may be confused with malignant orbital tumors. The diagnosis is usually made on the basis of the histological examination of a biopsy specimen harvested from the ocular tumor. However also other organ can be involved and must, therefore, be investigated in systemic sarcoidosis, as can be seen in pulmonary sarcoidosis.

The diagnosis should be definitely supported by the histological examination of biopsy specimens along with the detection of enlarged hilar lymph nodes, systemic and/or respiratory symptoms.

Furthermore, it is necessary to rule out other pathological conditions and diseases that may present as granulomatous diseases. In our case, sarcoidosis was diagnosed due to the presence of erythema nodosum, arthritis of both ankles, high serum ACE level and the detection of the typical noncaseating granulomas in the histological examination of the lymph node biopsy sample.

Corticosteroids should be considered as first-line treatment in orbital sarcoidosis. The surgical treatment is not recommended due to the likelihood of local recurrences and complications. In our patient, systemic corticosteroids were used and the orbital tumor was found to be completely resolved in the follow up examinations and radiologic imaging studies.

In conclusion, we diagnosed orbital sarcoidosis in a woman at a younger age than usual. Sarcoidosis should, therefore, be considered in patients presenting with orbital tumor.

Orbital sarcoidosis may occur in any portion of the eye and may present with a wide spectrum of symptoms, which may be the initial signs of systemic sarcoidosis. Early diagnosis and treatment may prevent a severe sarcoidosis-related loss of vision.

\section{REFERENCES}

1. Newman LS, Rose CS, Maier LA. Sarcoidosis. N Engl J Med. 1997; 336: 1224-34.

2. Hofmann S, Franke A, Fischer A, Jacobs G, Nothnagel M, Gaede KI, et al. Genome-wide association study identifies ANXA11 as a new susceptibility locus for sarcoidosis. Nat Genet. 2008; 40:1103-6.

3. Chen ES, Moller DR. Etiology of sarcoidosis. Clin Chest Med. 2008; 29: 365-77.

4. Smith G, Brownell I, Sanchez M, Prystowsky $\mathrm{S}$. Advances in the genetics of sarcoidosis. Clin Genet. 2008; 73: 401-12.

5. Kataria YP, Holter JF. Immunology of sarcoidosis. Clin Chest Med. 1997; 18: 719-39.

6. Kobak S, Sever F, Sivrikoz ON, Orman M. Sarcoidois: is it only a mimicker of primary rheumatic disease? A single center experience. Ther Adv Musculoskelet Dis. 2014; 6: 3-7.

7. Rothova A. Ocular involvement in sarcoidosis. Br J Ophthalmol 2000; 84: 110-6.

8. Obenauf CD, Shaw HE, Sydnar CF, Klintworth GK. Sarcoidosis and its ophthalmic manifestations. Am J Ophthalmol 1978; 86: 648-55.

9. Collison JMT, Miller NR, Green WR. Involvement of orbital tissues by sarcoid. Am J Ophthalmol 1986; 102: 302-7.

10. Hunter DG, Foster CS. Ocular manifestations of sarcoidosis. In: Albert DM, Jakobiec FA, eds. Principles and practice of ophthalmology. Philadelphia: WB Saunders; 1994. pp 443-450.

11. Bonfioli AA, Orefice F. Sarcoidosis. Semin Ophthalmol. 2005; 20: 177-82.

12. Jones NP. Sarcoidosis and uveitis. Ophthalmol Clin North Am. 2002; 15: 319-26.

13. Collison JM, Miller NR, Green WR. Involvement of orbital tissues by sarcoid. Am J Ophthalmol. 1986; 102: 302-7.

14. Henderson JW. Hystiocytic disorders. In: Henderson JW, ed. Orbital tumors. 3rd ed. New York, NY: Raven Press; 1994; 317-322.

15. Peterson EA, Hymas DC, Pratt DV, Mortenson SW, Anderson RL, Mamalis N. Sarcoidosis with orbital tumor outside the lacrimal gland: initial manifestation in 2 elderly white women. Arch Ophthalmol. 1998; 116: 804-6.

16. Holm E. A case of sarcoid of Boeck. Acta Ophthalmol Kbh. 1937; 15: 235-8.

17. King MJ. Ocular lesions of Boeck's sarcoid. Trans Am Ophthalmol Soc. 1939; 37: 442-58.

18. Benedict WL. Sarcoidosis involving the orbit. Arch Ophthalmol. 1949; 42: 546-50. 